\title{
Comunicación corta:
}

Solanum x curtilobum Juz. et Buk.: papa amarga cultivada con potencial para el mejoramiento genético

Bonifacio, A. 1; P. Ramos'; M. Alcon'; J. Gabriel'2

\section{Resumen}

Poco se conoce de la variabilidad intraespecífica de la especie Solanum $x$ curtilobum, de ahí que en el presente estudio se evaluó la fertilidad del polen, la viabilidad de la semilla sexual y la generación de variación genética y morfológica en materiales provenientes de semilla sexual de papa (SSP) de polinización libre de dos cultivares de papa amarga de la especie pentaploide $S$. $x$ curtilobum (Laram y Janq'u Ch'uqipitu) y una papa dulce tetraploide S.tuberosum ssp. andigena (Waych'a) como testigo. Los resultados mostraron porcentajes de germinación entre 61 a $68 \%$, demostrando la fertilidad del polen de los materiales evaluados. Así mismo, las plantas mostraron variación en el hábito de crecimiento, color de la flor, color de la piel del tubérculo, color de la pulpa del tubérculo, número y profundidad de ojos y forma de tubérculo. La ploidía fue variable, encontrándose progenies triploides, tetraploides y pentaploides en las familias evaluadas. Se observó rendimiento por planta individual de $398.1 \mathrm{~g} \pm \mathbf{4 2 8 . 9}$ y 558.9 g \pm 583.4 en progenies de Janq'u y Laram Ch'uqipitu respectivamente. La diversidad genética generada mediante SSP fue oportuna para la selección de genotipos para ampliar la diversidad genética.

Palabras claves adicionales:

Chuño, semilla sexual de papa, resistencia a heladas, ploidía.

Aceptado para publicación: 21 de marzo, 2013.

\footnotetext{
${ }^{1}$ Fundación PROINPA - Altiplano, Casilla 14709, La Paz, Bolivia.

${ }^{2}$ Fundación PROINPA -Valles Norte, Casilla 4285, Cochabamba, Bolivia.

E-mail: j.gabriel@proinpa.org .
} 


\section{Short Communication:}

Solanum x curtilobum Juz. et Buk.: A Bitter Potato

Cultivated with Potential for Plant Breeding

\section{Summary}

Little is known of intraspecific variability of the species $S$. $x$ curtilobum, hence in this study we evaluated the pollen fertility, seed viability and generating genetic and morphological variation were evaluated in true potato seed (TPS) from two open pollinated bitter potato cultivars of the Solanum $x$ curtilobum a pentaploid species (Laram and Janq'u Ch'uqipitu) and sweet potato of S.tuberosum ssp. andigena (Waych'a) a tetraploid species as control. The results showed germination rates from 61 to $68 \%$, demonstrating pollen fertility. The plants showed variation in growth habit, flower color, tuber skin color, tuber flesh color, number and depth of eyes and shape of tuber. The ploidy was variable, being triploid, tetraploid and pentaploid among progenies. Individual plant yield was observed from $398.1 \mathrm{~g} \pm 428.9$ and $558.9 \mathrm{~g} \pm 583.4$ in progenies of Janq'u and Laram Ch'uqipitu respectively. Genetic diversity generated by TPS was timely for the selection of genotypes to expand genetic diversity.

Additional key words:

Chuño, true potato seed, frost resistance, ploidy.

\section{Introducción}

La papa fue domesticada por las culturas andinas preincaicas que habitaron las zonas altas de Bolivia y Perú hace 14000 años a.C. (Morales, 2007). Actualmente forma parte de la cultura agrícola-alimentaria de la población asentada en el altiplano y valles interandinos, siendo un alimento básico de la población rural y urbana de ambos países. 
Bolivia, cuenta con una amplia diversidad de papas nativas de las ocho especies cultivadas conocidas (Estrada, 2000; Gabriel, 2010) que es apreciada por los productores e investigadores (mejoradores y curadores de germoplasma). La diversidad genética de la papa se conserva en bancos de germoplasma ex situ (Cadima et al., 2009) y por agricultores conservacionistas in situ (Terrazas et al., 2008).

El caso de la papa amarga, al parecer tiene importancia estratégica para los agricultores de escasos recursos económicos desde el punto de vista ecológico, alimenticio y económico, debido a que esta crece y produce en condiciones adversas de las alturas de los andes (Canahua, 1998). Se cultiva unas 13.000 ha anualmente en Bolivia que equivale al $11 \%$ de la superficie cultivada de papa en Bolivia (Thiele et al., 2008) Li y Palta (1977) y Bonifacio (1991), mencionan que la papa amarga tiene importancia por sus características de resistencia y/o tolerancia a heladas $\left(-4.8\right.$ y $-5.5^{\mathrm{a}} \mathrm{C}$.) y porque tienen propiedades para la elaboración del chuño y la tunta.

Lescano (1994) y Estrada (1991), mencionan que Solanum $x$ curtilobum, híbrido natural de $S$. $x$ juzepczukii y Solanum andigena, es una especie pentaploide $(2 \mathrm{n}=5 \mathrm{x}=60)$ con plantas de hábito semiarrosetado, de hojas erguidas y rígidas con foliolos angostos, corola rotácea y articulación del pedicelo muy próxima al cáliz.

Las papas amargas se caracterizan por presentar un alto contenido de glicoalcaloides en sus tubérculos, lo cual les confiere un sabor amargo. Los glicoalcaloides son metabolitos secundarios de la papa, importantes como mecanismo de defensa contra diferentes plagas, siendo también tóxicos para la alimentación animal y humana (Lachman et al., 2001).

Ochoa (2001) menciona que el hábitat de $S$. $x$ curtilobum es similar al de $S$. $x$ juzepczukii; es una especie que crece esencialmente en la Puna y se la cultiva desde las provincias de Huaraz y Bolognesi del departamento de Ancash (norte de Perú) hasta los departamentos de Cochabamba, La Paz, Oruro y Potosí en Bolivia, entre 3400 a 4100 msnm. Sin embargo, 
debido a su tolerancia a heladas y a la presencia de grandes concentraciones de poblaciones nativas Quechua y Aymara donde tienen gran popularidad, su mayor difusión se encuentra en el sur de Perú y en el altiplano peruano-boliviano. Ochoa (1979) y Schmiediche et al. (1980), mencionan que la variabilidad de $S$. $x$ curtilobum es escasa y está representada por dos morfotipos que varían por el color del tubérculo, pero no en base a las proteínas de reserva de los tubérculos. Los dos morfotipos fueron descritos por Lechnovich (1971) y corresponden a las formas china-malko y choque-pito.

Según Tapia y Fríes (2007), las fases fenológicas de la papa amarga son: la emergencia (a 35 días de la siembra), el inicio de estolones (a 20 días de la emergencia), el inicio de floración (a 25 días de emergencia), el inicio de tuberización (a 40 días de emergencia), la finalización de la floración (55 a 85 días de la emergencia) y la madurez fisiológica (a 145 días de emergencia).

En base a los conocimientos y saberes locales, la papa amarga Ch'uqipitu ( $S$. $x$ curtilobum) y la papa dulce Ajahuiri ( $S x$. ajanhuiri) son especies resistentes y/o tolerantes heladas. Sin embargo, ninguna de estas especies han sido incluidas en programas de mejoramiento genético de la papa en la zona andina (Gabriel et al., 2009).

Golmirzaie et al. (1990), mencionan que la multiplicación de papa por semilla sexual tiene varias ventajas, siendo las principales el bajo costo de producción y la escasa o nula transmisión de enfermedades. Estos mismos investigadores evaluaron seis progenies de polinización abierta de la especie Solanum tuberosum, encontrando un ligero incremento del rendimiento.

En los últimos años, como resultado de las preferencias del mercado, factores climáticos adversos, el empobrecimiento de los suelos y el incremento de plagas y enfermedades, han generado pérdida de parte de la diversidad genética heredada de nuestros antepasados. Adicionalmente, los cultivares de ciclo largo como las Ch'uqipitus podrían tener problemas de 
adaptación a las condiciones actuales, corriendo el riesgo de perderse.

El mejoramiento genético de la papa en Bolivia ha dado énfasis en la generación de cultivares comerciales aptas para la zona de valle, sub-trópico y partes altas de la cordillera oriental (Gabriel, 2010), siendo muy escasos los esfuerzos dedicados a las papas nativas del altiplano. La atención hacia las papas amargas es mucho más limitada o inexistente. Se conoce poco sobre la fertilidad de la especie $S$. $x$ curtilobum, la viabilidad de la semilla sexual y la generación de variación genética y morfológica a partir de la semilla sexual de esta especie.

El objetivo de la presente investigación fue evaluar la fertilidad del polen, la viabilidad de la semilla sexual y la variabilidad genética y morfológica de las progenies de dos papas amargas del grupo de las Ch'uquipitus (S. $x$ curtilobum) obtenidas de polinización libre.

\section{Materiales y métodos}

La investigación se realizó en los predios del Centro de Investigación Quipaquipani, Viacha, ubicada en la provincia Ingavi del Departamento de La Paz, Bolivia. Geográficamente se encuentra a 16ำ $40^{\prime} 30^{\prime \prime}$ de latitud sur, 68⒈ $68^{\prime \prime}$ ' de longitud oeste a una altura de de $3880 \mathrm{msnm}$, con precipitación media de $626.6 \mathrm{~mm}$ y una temperatura media de $10.1^{\circ} \mathrm{C}$.

El trabajo se desarrolló en dos fases.

Primera fase: Se eligieron dos cultivares amargos de $S . x$ curtibolum (Larama y Janq'u) ${ }^{3}$ y un cultivar nativo dulce de $S$. tuberosum ssp. andigena (Waych'a) (Tabla 1). Estos cultivares fueron sembrados en campo y manejados agronómicamente según las costumbres de la zona. No se hicieron autofecundaciones. Se dejó que las plantas tuvieran polinización libre. Se recolectaron las bayas (frutos) de las plantas madres. Luego de los frutos maduros se extrajo la

\footnotetext{
${ }^{3}$ Laram y Janq'u son palabras que vienen del aimara cuyos significados son "morado" y "blanco" respectivamente.
} 
semilla sexual, se las secó hasta $4 \%$ de humedad y se almacenó a $4^{\circ} \mathrm{C}$.

Tabla 1. Características de los cultivares utilizadas para obtener semilla sexual

\begin{tabular}{llcc}
\hline \multicolumn{1}{c}{ Cultivar } & \multicolumn{1}{c}{ Especie } & Ploidia & Características \\
\hline $\begin{array}{l}\text { Laram } \\
\text { Ch'uqipitu }\end{array}$ & $\begin{array}{l}\text { Solanum } x \\
\text { curtilobum }\end{array}$ & $2 n=5 \mathrm{x}=60$ & $\begin{array}{c}\text { Muy tardío, resistente } \\
\text { a heladas, amarga }\end{array}$ \\
Janq'o & $\begin{array}{l}\text { Solanum } x \\
\text { Ch'uqipitobum }\end{array}$ & $2 \mathrm{n}=5 \mathrm{x}=60$ & $\begin{array}{c}\text { Muy tardío, resistente } \\
\text { a heladas, amarga }\end{array}$ \\
Waych'a & $\begin{array}{l}\text { Solanum } \\
\text { tuberosum spp. } \\
\text { andigena }\end{array}$ & $2 \mathrm{n}=4 \mathrm{x}=48$ & $\begin{array}{c}\text { Tardío, dulce, buena } \\
\text { calidad culinaria }\end{array}$ \\
\hline
\end{tabular}

Segunda fase: La semilla sexual se almacigó en invernadero. Cuando las plántulas tenían dos hojas verdaderas fueron trasplantadas en maceta con sustrato apropiado (mezcla de tierra del lugar, arena y turba en una relación de 1:1:1). Las plántulas fueron mantenidas en macetas hasta alcanzar 8 a 10 $\mathrm{cm}$ de altura, luego fueron trasplantadas a campo a distancias de $0,75 \mathrm{~m}$ entre surcos y $0,4 \mathrm{~m}$ entre plantas. Se trasplantaron 18 familias de papa amarga con progenies por familia promedio de $28.9 \pm 28.2$ (mínimo por familia de 4 y máximo de 120 ) y una familia de papa dulce (Waych'a) sin ningún diseño experimental. Las progenies de las familias fueron enumeradas $y$ flanqueadas por un surco del progenitor materno.

Las variables de respuesta evaluadas fueron: el porcentaje de germinación, los caracteres agro-morfológicos de las plantas y tubérculos y rendimiento por planta. Estas variables fueron caracterizadas según los descriptores del CIP (Huaman et al., 1977). Empleando muestras de progenies de las familias, se ha realizado el conteo de cloroplastos para conocer preliminarmente el número de cromosomas de las progenies de semilla sexual según sugerencias de Orrillo y Bonierbale (2009). 


\section{Resultados y discusión}

Los dos cultivares de papa amarga pentaploides (Laram Ch'uqipitu y Janq'u Ch'uqipitu) tuvieron floración temprana, profusa y de larga duración, en cambio, el cultivar Waych'a tuvo floración tardía, profusa y de corta duración. La fructificación (cantidad de bayas) de las papas amargas fue abundante, al igual que del cultivar Waych'a (tetraploide). Esto mostró la alta fertilidad de los dos cultivares amargos.

En los cultivares amargos, las plantas provenientes del tubérculo - madre alcanzaron la madurez fisiológica a los 150 días; en cambio, en las plantas provenientes de semilla sexual alcanzaron la madurez a los 165 días después del trasplante en campo. Esto concuerda con lo reportado por Golmirzaie et al. (1990), quienes mencionaron que las plantas de semilla sexual maduran entre 15 a 20 días más tarde que la papa proveniente de tubérculos.

No hubo diferencias significativas al $\operatorname{Pr}<0,05$ de probabilidad en el porcentaje de germinación, denotándose un $68 \%, 66 \%$ y $61 \%$ para la Janq'u Ch'uqipitu, Laram Chu'qipitu y Waych'a respectivamente. Esto sugirió que la fertilidad del polen en los tres cultivares es similar, al igual que la viabilidad de la semilla sexual.

Los valores promedios y el intervalo de confianza para el porcentaje de germinación y sobrevivencia fueron altos (Tabla 2). Estos valores podrían considerarse promisorios para su uso a partir de semilla sexual para multiplicación y obtención de clones en programas de mejoramiento genético.

El porcentaje de prendimiento promedio de las plántulas fue de $84.5 \%$ con valor mínimo de $62 \%$ y máximo de $100 \%$, con un intervalo de confianza al 95\% de 79.4 - 89.6. La población de plantas enraizadas sufrieron algunas bajas, siendo el porcentaje de sobrevivencia promedio de $88.31 \%$ con valor mínimo de $75 \%$ y máximo de $100 \%$ con un intervalo de confianza al $95 \%$ de $84.1-92.5$. 
Tabla 2. Porcentaje de germinación y sobrevivencia de progenies de cultivares evaluados

\begin{tabular}{ccccc}
\hline Cultivar & \multicolumn{2}{c}{ Germinación } & \multicolumn{2}{c}{ Sobrevivencia } \\
\cline { 2 - 5 } & Promedio & I.C. 95\% & Promedio & I.C. 95\% \\
\hline Janq'o Ch'uqipitu & 68.33 & $59.29-77.37$ & 85.00 & $69.10-100.90$ \\
Laram Ch'uqipitu & 66.00 & $56.96-75.04$ & 77.33 & $57.43-93.23$ \\
Waych'a & 61.33 & $52.29-70.37$ & 87.67 & $71.77-103.57$ \\
\hline
\end{tabular}

I.C: Intervalo de confianza

A la cosecha se observó que no todas las plantas formaron tubérculos, estimándose que $94.7 \%$ de plantas formaron tubérculos con valor mínimo de $77 \%$ y máximo de $100 \%$, con un intervalo de confianza de $95 \%$ fue de 90.5 - 98.9. La ausencia de tuberización en algunas plantas probablemente se debió a deficiencias fisiológicas que condujeron a la inadaptación y/o a la segregación hacia formas no tuberosas. El elevado porcentaje de plantas obtenidas a partir de semilla sexual que formaron tubérculos, demostró que $S$. $x$ curtilobum generó plantas que tuberizan, ofreciendo una alternativa para la obtención de semilla-tubérculo a partir de semilla sexual.

La evaluación de los caracteres morfológicos en plantas provenientes de semilla sexual, permitió constatar una variación en el hábito de crecimiento, detectándose plantas erectas, decumbentes y postradas. Hubo escasa variación en el color de planta, siendo la mayoría de color verde con tallos de color púrpura y otras de color verde. Respecto del color de la flor, la variación fue entre violeta oscuro, violeta claro, rosada y blanca. La planta madre en ambos cultivares fue morada. Esto mostró la existencia de una variación genética en estos caracteres.

Con referencia a los tubérculos, se observó una amplia diversidad de colores en la piel de los tubérculos. Mientras la 
planta madre fue de color blanco o marrón claro, las progenies mostraron una amplia diversidad de colores entre y dentro de las familias evaluadas. En general, la papa - madre cuyo tubérculo fue de piel marrón claro, generó progenies con color marrón claro, amarilla, anaranjada, morada, violeta y rojo. La papa - madre de piel morada, generó progenies con tubérculos con piel de color morado, marrón oscuro, rojo claro, rojo oscuro y rosado oscuro. Esta variación en color de piel posiblemente se debe a la condición poliploide del cultivar Ch'uqipitu y a su constitución genética en heterocigosis, que hace que los cromosomas estén en nuliplex, simplex, dúplex, etc. tal como sostiene Mendoza (1990). El conteo de cloroplastos en las progenies de los cultivares Laram Ch'uqipitu y Janq'u Ch'uqipitu reportaron niveles de ploidía de triploide, tetraploide y pentaploide. Moreno-Perez et al. (2009) en triploides del género Tripsacum observaron variación en el número de cromosomas (21 a 33) en células meióticas, encontrando que la mayor frecuencia fue con 27 cromosomas (32.1\%) y que en muchas células se pierde un número variable de cromosomas durante la anafase de la división por meiosis.

Otros caracteres como la forma del tubérculo y la profundidad de los ojos mostraron también una amplia variación, observándose desde formas achatadas hasta alargadas.

Con referencia al color de la pulpa se observó en los tubérculos de las plantas madre de Laram Ch'uqipitu fueron de color de pulpa crema blanquecino, y las progenies variaron desde el amarillo, anaranjado, morado, rojo claro y rojo oscuro. De la misma manera se observó que los tubérculos de la planta madre de Janq'u Ch'uqipitu fueron de color blanco crema y su progenie varió desde el blanco, marrón, morado, amarillo y anaranjado.

Los resultados mostraron que existe variación genética a partir de semilla sexual en los cultivares pentaploides estudiados y esta información se podría extrapolar a otras especies de papa nativas. Esta forma de iniciar un proceso de selección puede ser una estrategia sencilla para generar variabilidad genética 
particularmente y obtener generar genotipos apropiados para la adaptación a cambios globales.

El número de tubérculos por planta fue variable en las progenies de semilla sexual. En las familias del cultivar Janq'u Ch'uqipitu, el número promedio de tubérculos fue $50.74 \pm 54.18$ con valor mínimo de 2 y máximo de 236 tubérculos por planta. En las familias del cultivar Laram Ch'uqipitu el número promedio de tubérculos fue de $69.47 \pm 75.1$ con valor mínimo de 3 y máximo de 426. El rendimiento promedio por planta en el cultivar Janq'u Ch'uqipitu fue $398.1 \mathrm{~g} \pm 428.9$ con rendimiento mínimo de $4 \mathrm{~g}$ y máximo de $2318 \mathrm{~g}$. Las progenies del cultivar Laram Ch'uqipitu fue de $558.9 \mathrm{~g} \pm 583.4$ con rendimiento promedio de $1 \mathrm{~g}$ y máximo de $2758 \mathrm{~g}$.

En un estudio reciente se caracterizó morfológica y molecularmente 1432 accesiones de seis especies cultivadas de la colección de papa cultivada boliviana, utilizando 42 marcadores microsatélite (Veramendi et al., 2013). Sobre la base de estos resultados se logró formar una colección núcleo de 270 accesiones que retiene un $100 \%$ de los alelos conservados. En este estudio de las 72 accesiones de $S$. $x$ cutilobum evaluados se conservó 33 accesiones en la colección núcleo, que equivale a $42 \%$ de las accesiones conservadas de la especie en la colección original. De igual manera Durán et al. (2002) encontraron que S. x curtilobum mostró diferencias principalmente en la profundidad de los ojos, color secundario de la pulpa y disección de hojas, aunque se observaron que las varianzas fueron estadísticamente diferentes en más variables (12 variables). Esto sugirió que los cultivares amargos de $S$. $x$ curtilobum, tienen una diversidad genética considerable, contrariamente a lo que indicado por Ochoa (1979) y Schmiediche et al. (1980). Así mismo, Rea (1992) y posteriormente Thiele et al. (2008), reportaron que el $15 \%$ de la producción total de papa en Bolivia corresponde a cultivares amargos y semi-amargos, lo cual demuestra su importancia en la economía y la alimentación local y nacional. Saravia (1999) encontró una diversidad importante de papas amargas en la zona de puna de la Prov. Tapacarí (Cochabamba), identificado dos especies de papas amargas $(S$. $x$ juzepczukii y $S . x$ 
curtilobum) y una especie semiamarga ( $S$. $x$ ajanhuiri). En esta zona alrededor del $70 \%$ de la producción de papa corresponde a los cultivares amargos y semi-amargos.

\section{Agradecimiento}

Se agradece el apoyo económico del Proyecto Mcknight en Bolivia.

\section{Bibliografía consultada}

Bonifacio, A. 1991. Germoplasma de papa amarga y caracterización preliminar en el altiplano boliviano. Páginas 27-32 En 1ra. Mesa Redonda Sobre papa amarga. La Paz, Bolivia.

Cadima, X.; F. Terrazas; A. Gandarillas. 2009. Los sistemas de conservación de recursos genéticos de Tubérculos y Raíces Andinas: La experiencia de PROINPA. Revista de Agricultura, Bolivia 43 (60): 31-36.

Canahua, A. 1998. Producción y promoción de semilla de papas amargas en Puno. CEDECUM-CIP, Puno, Perú. P.irr. 42 p.

Durán, G.; X, Cadima; J. Zeballo. 2003. Desarrollo de una colección núcleo de la colección de papa cultivada (Solanum ssp.) del banco de germoplasma de raíces y tubérculos andinos de Bolivia. Centro de Estadística Aplicada - CESA, UMSS, Bolivia: 1-7.

Estrada, N. 1991. Importancia genética de las papas amargas. Páginas 11-14 en Mesa Redonda Sobre papa amarga, 1ra. La Paz, Bolivia.

Estrada, N. 2000. La Biodiversidad en el Mejoramiento Genético de la papa. Bill Hardy, Emma Martínez (eds.) La Paz, Bolivia. 372 p.

Gabriel, J. 2010. Documento marco: Estrategias y perspectivas del mejoramiento genético de papa (Solanum tuberosum L.) en Bolivia. Fundación PROINPA, Cochabamba, Bolivia. 60 p.

Gabriel, J.; J. Magne; R. García; J. Coca. 2009. Variedades nativas de papa: Tecnología tangible para afrontar el efecto causado por la 
helada y la sequía. Revista Latinoamericana de la papa, Perú 15 (1):75-77.

Golmirzaie, A.; F. Serquen; R. Ortiz. 1990. Evaluación de tres generaciones de polinización libre en seis progenies de papa (Solanum tuberosum) proveniente de semilla (sexual). Revista Latinoamericana de la Papa, Perú 3(1):13-19.

Golmirzaie, A.; R. Ortiz; F. Serquen. 1990. Genética y mejoramiento de la papa por semilla sexual. CIP, Lima Perú. 35 p.

Huamán, Z.; J.T. Williams; W. Salhuara; L. Vincent. 1977. Descriptors for cultivated potato and for the maintenance and distribution of germplasm collection. International Board for Plant Genetic Resources, International Poatato Center, Rome, Italy. 43 p.

Lachman, J.; K. Hamouz; M. Orsak; V. Pivec. 2001. Potato glycoalkaloids and their significance in plant protection and human nutrition - review. Series Rostlinná Výroba, Czechoslovakia 47 (4): 191.

Lescano, J.L. 1994. Genética y Mejoramiento de cultivos alto andinos, ed. Primera edición, edit. Cima, La Paz Bolivia, p. 208 - 210.

Lechnovich, V.S. 1971. Classification of cultivated species. Page 41304 in S.M. Bukasov, Ed. Flora of cultivated plants, Vol. IX. The Potato, Leningrad: Publ. House Colos.

Li, P. ; J. Palta. 1977. Frost killing temperatures of 60 tuber-bearing Solanum species. Amer. Potato J. 54: 452-456.

Mendoza, H. 1990. Avances en el mejoramiento genético de la papa en los países del Cono Sur. Páginas 133 - 148. In: Hidalgo, O.A. y

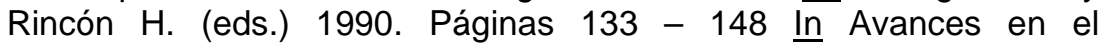
mejoramiento genético de la papa en los países del Cono Sur. CIPINTA-PROCIPA.

Morales, F. 2007. Sociedades precolombinas asociadas a la domesticación y cultivo de la papa (Solanum tuberosum) en Sudamérica. Revista Latinoamericana de la Papa, 14 (1): 1-9.

Moreno-Perez, E.; A. García-Velásquez; C.H. Aendano-Arrazate. 2009. Cytological study in diploid and polyploid populations of the Tripsacum genus. J. Interciencia, México 34 (11): 791-795. 
Ochoa, C. 1979. Review of recommendations of March 1976 Conference: Status of Solanum expedition, collections, and classifications. Page 23-25 In Report of the Planning Conference of the exploration, taxonomy, and Maitenance of Potato Germplasm III. Lima, Perú, International Potato center.

Ochoa, C. 2001. Las papas de Sudamérica: Bolivia. Plural editores/CID, La Paz, Bolivia. 535 p.

Orrillo, M. ; M. Bonierbale. 2009. Manual Técnico: Biología reproductiva y citogenética de la papa. Red LatinPapa-CIP. 22 p.

Rea, J. 1992. Vigencia de las papas nativas en Bolivia. Página 111 in La papa amarga. Primera mesa redonda: Perú - Bolivia. 7-8 Mayo 1992. ORSTOM, La Paz, Bolivia.

Saravia, G. 1999. Biodiversidad en papas amargas. http://www.condesan.org/e-foros/insitu99/Saravia\%20(spanish).html (Consulta: 26 de agosto, 2012).

Schmiediche, P.; J. Hawkes; C. Ochoa. 1980. Breeding of the cultivated potato species Solanum juzepczukii and S. x cutilobum Juz. And Buk. I. A study of the natural variation of $S$. $x$ cutilobum and their wild progenitors S. acaule Bitt. Euphytica, Nedherlans 29 (3): 685-704. Tapia, M.; M. Fries. 2007. Guía de campo de los cultivos andinos, Perú p.73-75.

Terrazas, F.; X. Cadima; R. García; J. Zeballos. 2008. Catálogo etnobotánico de papas nativas. Tradición y cultura de los Ayllus del Norte Potosí y Oruro. Ricerca \& Cooperazione, Unión Europea, Centro de Apoyo a Desarrollo, GTZ, Fundación PROINPA, MDRyMA. Cochabamba - Bolivia. $189 \mathrm{p}$.

Thiele, G.; G. Hareau; V. Suarez; E. Chujoy; M. Bonierbale; L. Maldonado. 2008. Varietal change in potatoes in developing countries and the contribution of the International Potato Center: 1972-2007. International Potato Center (CIP), Lima, Peru. Working Paper 2008-6. $46 \mathrm{p}$.

Veramendi, X. Cadima; J. Gabriel. 2013. Integración molecular y morfológica para la formación de la Colección Núcleo de papa en Bolivia. Revista Latinoamericana de la Papa (Aceptado para publicación Vol.17-2, 2013). 